\title{
PROS AND CONS FOR FLUORESCENT IN SITU HYBRIDIZATION, KARYOTYPING AND NEXT GENERATION SEQUENCING FOR DIAGNOSIS AND FOLLOW-UP OF MULTIPLE MYELOMA
}

\author{
Ikbal Atli E ${ }^{1, *}$, Gurkan $\mathrm{H}^{1}$, Onur Kirkizlar $\mathrm{H}^{2}$, Atli E ${ }^{1}$, Demir $\mathrm{S}^{1}$, Yalcintepe $\mathrm{S}^{1}$, Kalkan $\mathrm{R}^{3}$, Demir $\mathrm{AM}^{2}$ \\ *Corresponding Author: Assistant Professor Emine Ikbal Atli, Department of Medical Genetics, Faculty \\ of Medicine, Trakya University, Balkan Campus, Highway D100, Edirne, Turkey 22030. Tel: +284-235- \\ 76-41/23-30. Fax:+284-235-86-52. E-mail: eikbalatli@trakya.edu.tr
}

\begin{abstract}
Multiple myeloma (MM) is one of the plasma cellrelated hematological malignancies exceeding $10.0 \%$ of all marrow cells, and they make a paraprotein that is a marker of the disease. Myeloma is one of the most common types of hematological malignancies in humans. Genetic biomarkers have been used for prognostic markers in patients diagnosed with MM. The genetic and genomic changes have been identified using karyotyping, fluorescent in situ hybridization (FISH), next generation sequencing (NGS), specifically whole-genome sequencing or exome sequencing. Circulatory plasma cells, circulating free DNA (cfDNA) and microRNAs (miRNAs) comprised in liquid biopsy are potentially used in diagnosis/prognosis of MM. In this study, we analyzed and compared results of karyo-typing, FISH and NGS in 35 MM cases. Diagnostic strategies are expanding rapidly and newly developed NGS-based testing may help the understanding of the complexities of genetic alterations in karyotypically normal cases.
\end{abstract}

Keywords: Cytogenetics; Fluorescent in situ hybridization (FISH); Multiple myeloma; Next generation sequencing (NGS).

\section{INTRODUCTION}

Multiple myeloma (MM) originates from the neoplastic transformation and proliferation of B-cells [1]. Multiple myeloma is a kind of plasma cell malignancy that is characterized by the accumulation of clonal plasma

\footnotetext{
${ }^{1}$ Department of Medical Genetics, Faculty of Medicine, Edirne, Trakya University, Edirne, Turkey

${ }^{2}$ Department of Hematology, Faculty of Medicine, Trakya University, Edirne, Turkey

${ }^{3}$ Department of Medical Genetics, Faculty of Medicine, Near East University, North Nicosia, Northern Cyprus
}

cells in the bone marrow [2]. Monoclonal gammopathy of undetermined significance (MGUS) is a heterogeneous disorder that starts with premalign stage and progresses to bone destruction, suppression of bone marrow function and renal failure $[3,4]$. Identification of cytogenetic abnormalities and development of novel prognostic biomarkers are the important cornerstones of MM in recent years [2,5].

The genetic background of MM is complex and contains several translocations [6]. Conventional cytogenetic analysis should be included in the initial diagnostic tests for patients suspected of carrying MM. Cytogenetic abnormalities have a prognostic significance in $\mathrm{MM}$, and approximately $30.0-50.0 \%$ of cases demonstrate abnormal karyotypes, and abnormality frequency is also decreased in newly diagnosed patients. Cytogenetic analysis can provide useful prognostic information but particularly low spontaneous proliferative activity of tumor cells in the early stage of the disease is considered to be an important limiting factor [7]. Interpretation of abnormal metaphase cells may be difficult due to the poor quality of metaphase spreads and chromosomal morphologies, and this situation prevents identification of critical chromosomal changes in MM patients [8]. These limitations have been partially overcome using molecular cytogenetic techniques such as fluorescence in situ hybridization (FISH) and comparative genomic hybridization (CGH) [2]. The important point of FISH analysis is target specificity and CGH is not suitable for identification of balanced abnormalities [7].

Currently, in standard usage, conventional chromosome analysis, targeted FISH panels, and single nucleotide polymorphism (SNP) microarrays, are used to detect chromosome translocations and gains and losses in MM. Moreover, the development of next generation sequencing (NGS) expanded our knowledge of MM biology, identifying new and recurrent driver abnormalities such as single nucleotide variants (SNVs) and deletions [6]. In this study, we aimed to provide a comparison of both conventional 
cytogenetic/FISH results and NGS in Turkish patients who have been newly diagnosed to carry MM.

\section{MATERIALS AND METHODS}

The present study includes 35 patients (17 males and 18 females) from April 2018 to December 2019. Written informed consent was obtained from all subjects after a full explanation of the study protocol, which was approved by the Ethics Committee of the Faculty of Medicine, Trakya University, Erdine, Turkey, and conducted in accordance with the ethics principles established by the Declaration of Helsinki.

Cytogenetic Evaluation. Cytogenetics studies were carried out either on bone marrow or peripheral blood of patients. Diagnoses were based on the International Myeloma Working Group (IMWG) definition of MM [5]. The morphology of patient bone marrow specimens confirmed a myeloma in all cases.

Chromosomal analysis was performed on cultured bone marrow samples using a standard G-banding technique. At least 20 metaphase spreads were analyzed and results were reported according to the International System for Human Cytogenetic Nomenclature (2016) [9].

Fluorescent In Situ Hybridization Analysis. In order to analyze molecular cytogenetic abnormalities, inter phase FISH was applied to non dividing cells, according to the manufacturer's instructions (Cytocell, Cambridge,
Cambridgeshire, UK). The list of analyzed loci is as follows: IGH/FGFR3 Dual Fusion (DF) Probe (Cytocell) for $(4 ; 14)$ (p16.3; q32.33); IGH/MAF DF Probe (Cytocell) for $\mathrm{t}(14 ; 16)$ (q32.33; q23.1); IGH/CCND1/MYEOV DF Probe (Cytocell) for t(11;14)(q13;q32.33); 13q14.3 Dual Color Probe (Cytocell) for del(13q14); P53 (TP53) Dual Color Probe (Cytocell) for del(17p13); ATM Dual Color Probe (Cytocell) for del(11q22.3).

The evaluation of FISH signals was performed using a fluorescence microscope (Axio Imager, M1; Carl Zeiss, Göttingen, Germany) with the software Cytovision 3.6 (Leica Biosystems Inc., Buffalo Grove, IL, USA). At least 200 inter phase nuclei were analyzed for each slide.

Next Generation Sequencing. Next generation sequencing technology allows millions of sequence readings to be processed in parallel; this makes it an excellent tool for identification of low-rate clonality detection. In fact, this technology is used not only to cover large genomic regions, but also for ultra-deep sequencing of small genomic regions, so it can be used for assessment of clonal rearrangements of MM-related markers such as the immunoglobulin heavy chain locus $(\operatorname{IgH})$ gene [10].

DNA was isolated from bone marrow (QIAamp DNA Blood Mini Kit; Qiagen GmbH, Hilden, Germany) and quantified using a Qubit fluorometer (Thermo Fisher Scientific, Waltham, MA, USA). Multiple myeloma specific target genes were selected as shown in Table 1. Myeloid

Table 1. List of diseases and genes covered in the next generation sequencing analysis.

\begin{tabular}{|c|c|}
\hline Disease & Genes Covered \\
\hline Acute lymphoblastic leukemia (ALL) & $\begin{array}{l}\text { ASXL2, ATM, BRAF, CALR, CDKN2A, CREBBP, CRLF2, CSF3R, CTCF, DNM2, } \\
\text { EGFR, EP3OO, FBXW7, GATA2, HNRNPK, HRAS, IKZF3, IL7R, KDM6A, KDR, } \\
\text { KMT2C, LRRC4, MAP2K1, MLH1, MSH2, MSH6, NOTCH1 NTRK3, PAX5, } \\
\text { PDGFRA, PMS2, PRAMEF2, PTEN, RELN, SMARCB1 }\end{array}$ \\
\hline Acute myeloid leukemia (AML) & $\begin{array}{l}\text { ANKRD26, ASXL1, ATM, BCOR, BCORL1, BIRC3, BRAF, C17orf97, CALR, } \\
\text { CARD11, CBLC, CDKN2A, CEBPA, CHEK2, CREBBP, CSF1R, CSF3R, CTCF, } \\
\text { DAXX, DDX41, DNM2, DNMT1, ELANE, EP3O0, FLRT2, FLT3, GATA1, GATA2, } \\
\text { HNRNPK, IDH1, IDH2, IKZF1, IL7R, JAK1, JAK3, KDM6A, KDR, KIT (CD117), } \\
\text { KMT2A, KMT2C, KRAS, LRRC4, MAP2K1, MPL, MSH6, MYC, NBN, NOTCH1, } \\
\text { NPM1, NRAS, NSD1, NTRK3, OR13H1, OR8B12, P2RY2, PCDHB1, PDGFRA, } \\
\text { PHF6, PRAMEF2, PRPF8, PTEN, PTPN11, RAD21, RUNX1 (AML1), SF1, SF3A1, } \\
\text { SMARCB1, SMC1A (SMC1L1), SMC3, SRP72, SRSF2, STAG2, STXBP2, U2AF1, } \\
\text { U2AF2, WT1 }\end{array}$ \\
\hline Chronic lymphocytic leukemia (CLL) & $\begin{array}{l}\text { ADA, BIRC3, BLM, BRAF, CALR, CHEK2, CSF3R, KCNA4, KLHL6, KMT2C, } \\
\text { MAP2K1, NBN, NPAT, NTRK3, OR13H1, OR8B12, PRAMEF2, SRP72, TAL1, TERT, } \\
\text { TUBA3C, WAS, WRN }\end{array}$ \\
\hline Chronic myeloid leukemia (CML) & $\begin{array}{l}\text { ABL1, CALR, CDKN2A, CEBPA, CREBBP, CSF1R, CSF3R, FBXW7, GATA2, } \\
\text { KDM6A, MSH2, MSH6, RB1, SMC1A (SMC1L1),TP53 }\end{array}$ \\
\hline Chronic myelomonocytic leukemia (CMML) & CALR, CEBPA, CSF1R, CSF3R, HRAS, KMT2C, LUC7L2, SRSF2 \\
\hline Chronic neutrophilic leukemia (CNL) & CALR, CSF3R \\
\hline Multiple myeloma (MM) & $\begin{array}{l}\text { ATM, BCL6, BCR, BIRC3, BRAF, CDKN2A, CEBPA, EGFR, FBXW7, GJB3, HRAS, } \\
\text { KDM6A, MYC, NOTCH1, PTEN, SH2D1A, SMARCB1 }\end{array}$ \\
\hline Myelodysplastic syndromes (MDS) & $\begin{array}{l}\text { ATRX, CALR, CDKN2A, CEBPA, CSF1R, CSF3R, EP300, ETNK1, GNAS, HRAS, } \\
\text { KDM6A, KMT2A, KMT2C, RAD21, RB1, SETBP1, SF1, SF3A1, SMC3, SRSF2, } \\
\text { STAG2, U2AF1, U2AF2, XPO1, ZRSR2 }\end{array}$ \\
\hline
\end{tabular}


Panel QIAseq Targeted DNA Panel (Qiagen GmbH) was used to be able to enrich genes and construct libraries for NGS analysis for 141 genes that are commonly mutated in MM. This panel covers exonic regions of genes and $\pm 5-10$ bases of exon/intron boundaries. The panel was designed to cover genes based on library fragments of 250 bp produced from high-quality DNA samples (Table 1). Amplified targets were sequenced on the NextSeq (Illumina Inc., San Diego, CA, USA) sequencer according to the manufacturer's protocol for paired-end sequencing.

The ingenuity variant analysis (IVA) program was used for data analysis and quality assessment of SNVs, short insertions and deletions. Multiple databases were consulted when performing variant analysis of patients [American College of Medical Genetics and Genomics (ACMG) (www.acmg.net), Bethesda, MD, USA [11]; COSMIC (https://cancer.sanger.ac.uk/cosmic); HGMD (www.hgmd.cf.ac.uk.ac.index.php); ClinVar (www.ncbi. nlm.nih.gov.clinvar/); Uniprot (www.uniprot.org/); Genom AD (https//gnomad.broadinstitute.org/); Varsome (https:// varsome.com); Franklin (https://flanklin.genoox. com/clinical-db/home)]. Amplicons were considered as dropout and excluded from analysis if the coverage at any analyzed position in any of the two-paired end sequences (minimal coverage) was $<80$ reads. Validity of somatic mutations was checked against publicly accessible databases. Variants detected at a coverage of $>100 \times$, with allele frequency $>5.0 \%$ were included for subsequent investigation. Known hotspots or clinically actionable variants detected below these thresholds were verified using orthogonal methods such as Sanger sequencing.

\section{RESULTS}

Thirty-five patients with MM were studied. Conventional cytogenetic analysis failed in 10 (28.0\%) out of 35 patients. Twenty-five $(71.4 \%)$ out of the $35 \mathrm{MM}$ patients were reported to have a normal karyotype. In order to identify trisomies/monosomies, del13 and 17p13.1, and trans-locations involving the immunoglobulin heavy chain locus (IgH) and immunoglobulin $\lambda$-light chain locus (IgL- $\lambda$ ), FISH was performed. Abnormal FISH results were revealed in eight (22.8\%) out of 35 cases (Table 2 ).

In our series, 18 patients were female and 17 were male (sex ratio 1:1). The ages of patients ranged from 35 to 84 years at diagnosis, with a median age of 65 . The frequency of molecular cytogenetic abnormalities and molecular variations in patients with abnormal FISH/NGS results are summarized in Table 3.

Next generation sequencing analysis was applied to all cases and pathogenic or likely pathogenic variations were detected in six out of the 25 cytogenetically normal
Table 2. Frequencies of genetic abnormalities in patients who were evaluated with conventional cytogenetic/ fluorescent in situ hybridization and next generation sequencing.

\begin{tabular}{|l|c|}
\hline Cytogenetic Abnormalities & $\boldsymbol{n}(\%)$ \\
\hline No metaphase & $10(28.00)$ \\
\hline Normal karyotype & $25(71.40)$ \\
\hline FISH Abnormalities & \\
\hline$-13 /$ del(13q) & $3(8.57)$ \\
\hline$-17 /$ del(17p) & $2(5.71)$ \\
\hline $\mathrm{t}(11 ; 14)(\mathrm{q} 13 ; \mathrm{q} 32) / / g H-C C N D 1$ & $2(5.71)$ \\
\hline $\mathrm{t}(4 ; 14)(\mathrm{p} 16 ; \mathrm{q} 32) / I g H-F G F R 3$ & $2(5.71)$ \\
\hline $\mathrm{t}(14 ; 16)(\mathrm{p} 32 ; \mathrm{q} 23) / I g H-M A F$ & - \\
\hline Chromosome 1 amplification $(C K S 1 B / C D K N C$ gene $)$ & $4(11.42)$ \\
\hline NGS Results & \\
\hline Pathogenic variations & $10(28.57)$ \\
\hline Likely pathogenic variations & $3(8.57)$ \\
\hline Variants of unknown significance (VUS) & $11(31.42)$ \\
\hline
\end{tabular}

$n$ : number; FISH: fluorescent in situ hybridization; NGS: next generation sequencing.

cases. Additionally, a pathological variant was identified in five out of eight cases who had abnormal FISH results. Variants of unknown significance (VUS) were identified in 10 cases and the sex ratio was detected as 1.3 (F:M, $13 / 10$ ) in all of the abnormal cases.

\section{DISCUSSION}

Genetic abnormalities hold an important place to be able to risk stratification and the risk adapted treatment strategies in MM [5,12]. Conventional cytogenetics, FISH or microarray-based technologies are still golden standard for identification of genetic abnormalities in MM [10]. Even though conventional cytogenetic analysis is an efficient approach, providing information about whole chromosome status within a single experiment [13], but a low mitotic index of plasma cells is the major problem of MM cases which directly affects conventional cytogenetic studies. In our study, conventional cytogenetic analysis was successfully performed in $71.4 \%$ (25/35) of samples. The rate of outcome with conventional cytogenetics in MM patients varies between 29.5 and $64.0 \%$ in the literature $[14,15]$. Our success rate was higher than the previously described range even without using a specific stimulant for plasma cells.

The identification of the cryptic translocations is an important component of testing strategies in MM. Therefore, FISH is a suitable technique for evaluation of these diagnostic and prognostic cryptic translocations in MM. Despite the advantages and disadvantages of all of these techniques, we performed all of them in this study and we 
Table 3. Comparison of next generation sequencing and fluorescent in situ hybridization/karyotype results.

\begin{tabular}{|c|c|c|c|c|}
\hline $\begin{array}{l}\text { Sex-Age } \\
\text { (years) }\end{array}$ & \multicolumn{2}{|c|}{ Pathogenic Variations } & FISH Abnormalities & $\begin{array}{l}\text { Cytogenetic } \\
\text { Evaluation }\end{array}$ \\
\hline $\mathrm{M}-70$ & \multicolumn{2}{|l|}{ NRAS: c. $181 \mathrm{C}>\mathrm{A}$} & normal & $46, X Y$ \\
\hline F-74 & \multicolumn{2}{|l|}{ NRAS: c. $183 \mathrm{~A}>\mathrm{T}$} & CDKN2C;CKS1B $\times 3 ;$ IgH/FGFR3+ & - \\
\hline M-41 & \multicolumn{2}{|l|}{ normal } & D13S25; 13qter; RB1 $1 \times 1 ; C K S 1 B \times 3$; IgH/FGFR3+ & $46, X Y$ \\
\hline F-67 & \multicolumn{2}{|l|}{ NRAS: c. $181 \mathrm{C}>\mathrm{A}$} & CKS1B; CCND1; P53; CEP17×3 & $46, X X$ \\
\hline M-58 & \multicolumn{2}{|c|}{ DNMT3A: c. $2645 \mathrm{G}>\mathrm{A}$} & normal & $46, X Y$ \\
\hline M-74 & \multicolumn{2}{|c|}{ normal } & $C K S 1 B \times 4$ & $46, X Y$ \\
\hline M-61 & $\begin{array}{l}\text { SF3B1: c. } 1866 \mathrm{G}>\mathrm{T} \\
\text { TP53: c. } 818 \mathrm{G}>\mathrm{A}\end{array}$ & $\begin{array}{l}\text { VUS } \\
C B L B: \text { c. } 1472 \mathrm{~A}>\mathrm{G}\end{array}$ & $R B 1 ; D 13 S 25 \times 1$ & $46, X Y$ \\
\hline M-68 & \multicolumn{2}{|c|}{ TNFRSF13B: c.310T $>\mathrm{C}$} & normal & - \\
\hline F-71 & \multicolumn{2}{|c|}{ TET2: c.3543_3544delCT } & $\mathrm{P} 53 \times 1$ & $46, X X$ \\
\hline M-69 & \multicolumn{2}{|c|}{ ATM: c. $2250+2 \mathrm{~T}>\mathrm{A}$} & $R B 1 ; D 13 S 25 \times 1$ & $46, X Y$ \\
\hline F-67 & \multicolumn{2}{|l|}{ normal } & $C C N D 1 \times 3 ; C K S 1 B \times 4$ & - \\
\hline M-64 & ASXL1: c. $1141 \mathrm{G}>\mathrm{T}$ & $\begin{array}{l}\text { likely pathogenic } \\
\text { FLT3: c. } 2678 \mathrm{C}>\mathrm{T}\end{array}$ & normal & $46, X Y$ \\
\hline F-70 & & $K R A S:$ c. $183 \mathrm{~A}>\mathrm{C}$ & normal & - \\
\hline F-76 & & TERT: c.2035T $>\mathrm{G}$ & normal & - \\
\hline F-63 & & $\begin{array}{l}\text { VUS } \\
A K A P 13 \text { : c. } 7265 \mathrm{G}>\mathrm{A}\end{array}$ & normal & - \\
\hline F-52 & & MYD88: c.815G>A & normal & - \\
\hline F-66 & & CBLB: c. $2434 \mathrm{G}>\mathrm{A}$ & normal & $46, \mathrm{XX}$ \\
\hline F-67 & & BLM: c.2237C $>\mathrm{T}$ & normal & $46, X X$ \\
\hline F-58 & & $\begin{array}{l}C B L B: \text { c. } 1927 \mathrm{~A}>\mathrm{G} \\
C S F 3 R: \text { c. } 355 \mathrm{G}>\mathrm{A}\end{array}$ & normal & $46, X X$ \\
\hline F-49 & & SF3A1: c.458T $>$ C & normal & $46, X X$ \\
\hline M-84 & & SF3B1: c. $422 \mathrm{~A}>\mathrm{G}$ & normal & - \\
\hline M-50 & & ASXL1: c. $143 \mathrm{G}>\mathrm{T}$ & normal & $46, X Y$ \\
\hline F-72 & & $M P L:$ c. $1481 \mathrm{~T}>\mathrm{G}$ & normal & $46, X X$ \\
\hline
\end{tabular}

FISH: fluorescent in situ hybridization; M: male; F: female.

obtained different results from these techniques. Although karyotyping is still the golden standard for hematological malignancies, the results are not always beneficial for clinical usage due to the limitations of the chromosomal resolution. Fluorescent in situ hybridization is one of the user-friendly and low-cost tests for prevention of resolution limit of MM cases. High accuracy results are obtained with frequent translocations (those accompanying the IgH locus and others) and probes used specifically for deletion/ amplifications. However, FISH does not always allow low clonal cell detection for minimal residual disease (MRD). The other problem is that FISH probes are less sensitive for detecting unbalanced translocations, and another technical problem is adjusting of the cutoffs for various FISH probes. In some laboratories, cutoffs are described on a regular basis and adjust them as a $>3$ SD [13]. However, it is not clear if these abnormalities have the same prognostic significance when present at low levels at diagnosis. In both instances, it is important to understand the limitations of the FISH assay when interpreting and reporting the results. Despite its pros and cons, FISH and karyotyping are still the golden standard for diagnosis and follow-up of the disease. Since 2016, several publications have been reported related with NGS usage in MM. Published studies focused on sequencing of the patients' exome during the time of diagnostic reading and relapse. These studies also confirm the molecular heterogeneity of MM. The KRAS and NRAS genes (approximately 25.0 and $20.0 \%$ of patients, respectively) are the most frequently mutated genes in MM. The prognostic information of these mutations is still limited. Until now, detrimental prognostic value of mutations has only been shown for TP53 mutations (observed in 6.0-8.0\% of patients) and other rare mutations (present in $<3.0 \%$ of the patients) may affect the outcome of the disorder [10].

Next generation sequence testing is suitable during the low plasma cell percentage in the blood or bone marrow. Next generation sequencing is very useful in the case of low mitotic index, which cannot give permission for classical methods to be able to identify the abnormalities. The major limitation of NGS is not being used for covering broad genomic regions and the ultra-deep sequencing of small genomic regions, but it is applicable for detection of the 
MM-associated clonal rearrangements of the $\mathrm{IgH}$ genes that are molecular markers for MRD evaluation. The complex nature of the MM-related genes makes them a target for the NGS approach. In this study, we identified pathological variations in six cases that were reported normal using conventional techniques. This showed us that the efficiency of FISH and karyotype analyses are limited, and showed the diagnostic and prognostic importance of NGS panels with frequent variants in the myeloid series. Next generation sequencing assays should be used in clinical practice due to the sensitivity of $10^{-6}$ or because they are evaluated with higher sensitivity and less expensive than other technologies such as digital polymerase chain reaction (ddPCR) [6,10].

As a result, technological developments are important in the classification of MM at the molecular level. It is inevitable to perform both traditional cytogenetic and FISH applications to reveal new recurrent specific chromosomal abnormalities or other genetic abnormalities in MM patients. Technologies such as NGS are an emergency tool to determine the heterogeneous nature of the disease and to determine the true clinical stage of the disease.

Acknowledgments. We would like to thank all the patients who participated in this study.

Declaration of Interest. The authors report no conflicts of interest. The authors alone are responsible for the content and writing of this article.

\section{REFERENCES}

1. Bolli N, Biancon G, Moarii M, Gimondi S, Li Y, de Philippis C, et al. Analysis of the genomic landscape of multiple myeloma highlights novel prognostic markers and disease subgroups. Leukemia. 2018; 32(12): 2604-2616.

2. Li S, Lim HH, Woo KS, Kim SH, Han JY. A retrospective analysis of cytogenetic alterations in patients with newly diagnosed multiple myeloma: A single center study in Korea. Blood Res. 2016; 51(2): 122-126.

3. Rajkumar SV. Multiple myeloma: 2014 Update on diagnosis, risk-stratification, and management. Am J He-matol. 2014; 89(10): 999-1009.

4. Talley PJ, Chantry AD, Buckle CH. Genetics in myeloma: Genetic technologies and their application to screening approaches in myeloma. Br Med Bull. 2015; 113(1): 15-30.

5. Munshi NC, Anderson KC, Bergsagel PL, Shaughnessy J, Palumbo A, Durie B, et al. Consensus recommendations for risk stratification in multiple myeloma: Report of the International Myeloma Workshop Consensus Panel 2. Blood. 2011; 117(18): 4696-4700.
6. Yellapantula V, Hultcrantz M, Rustad EH, Was-serman E, Londono D, Cimera R, et al. Comprehensive detection of recurring genomic abnormalities: A targeted sequencing approach for multiple myeloma. Blood Cancer J. 2019; 9(12): 101-109.

7. Aydin C, Ulas T, Hangul C, Yucel OK, Iltar U, Salim $\mathrm{O}$, et al. Conventional cytogenetics and interphase fluorescence in situ hybridization results in multiple myeloma: A Turkey laboratory analysis of 381 cases. Indian J Hematol Blood Transfus. 2020; 36(2): 284291. doi: 10. 1007/s12288-019-01215-5. Epub 2019 Oct 25.

8. Fonseca R, Barlogie B, Bataille R, Bastard C, Bergsagel PL, Chesi M, et al. Genetics and cytogenetics of multiple myeloma: A workshop report. Cancer Res. 2004; 64(4): 1546-1558.

9. McGowan-Jordan J, Simons A, Schmid M, editors. An International System for Human Cytogenomic Nomenclature (ISCN 2016). Basel, Switzerland: Karger, 2016.

10. Romano A, Palumbo GA, Parrinello NL, Conti-cello C, Martello M, Terragna C. Minimal residual disease assessment within the bone marrow of multiple myeloma: A review of caveats, clinical significance and future perspectives. Front Oncol. 2019; 9(20): 699.

11. Richards S, Aziz N, Bale S, Bick D, Das S, GastierFoster J, et al. Standards and guidelines for the interpretation of sequence variants: A joint concensus recommendation of the American College of Medical Genetics and Genomics and the Association of Molecular Pathology. Genet Med. 2015; 17(5): 405-424. doi: 10. 1038/gim. 2015.30.

12. Kapoor P, Fonseca R, Rajkumar SV, Sinha S, Gertz MA, Stewart AK, et al. Evidence for cytogenetic and fluorescence in situ hybridization risk stratification of newly diagnosed multiple myeloma in the era of novel therapies. Mayo Clin Proc. 2010; 85(6): 532-537.

13. Saxe D, Seo EJ, Bergeron MB, Han JY. Recent advances in cytogenetic characterization of multiple myeloma. Int J Lab Hematol. 2019; 41(1): 5-14.

14. Kaufmann H, Ackermann J, Greinix H, Nösslinger T, Gisslinger H, Keck A, et al. Beneficial effect of highdose chemotherapy in multiple myeloma patients with unfavorable prognostic features. Ann Oncol. 2003; 14(11): 1667-1672.

15. Durak BA, Akay OM, Sungar G, Bademci G, Aslan $\mathrm{V}$, Caferler J, et al. Conventional and molecular cytogenetic analyses in Turkish patients with multiple myeloma. Turk J Haematol. 2012; 29(2): 135-142. 